\title{
The effect of reversal shifts and scrambled shock on preference for signaled shock established with unscrambled shock
}

\author{
BRIAN M. KRUGER and PATRICK E. CAMPBELL \\ Wright State University, Dayton, Ohio 45435 \\ and \\ MARK S. CRABTREE \\ Systems Research Laboratories, Inc., Dayton, Ohio 45440
}

\begin{abstract}
Four rats were given free choice of sides in a shuttlebox. Each rat was enclosed in each side for $30 \mathrm{~min}$ prior to each daily 1 -h choice session. Shocks of 2 sec duration were administered on both sides at irregular intervals. Shocks were preceded by a tone signal on one side. All rats showed a reliable preference for the signaled shock side in the first phase. Reversal of the visual stimuli differentiating the signal side from the nonsignal side eliminated the preference for all rats. Reinstatement of the original relations resulted in the reestablishment of the preference for two rats. In the next phase, changing to scrambled shock from the previously used unscrambled shock eliminated the preference for these two rats. The preference was not reestablished when unscrambled shock was again used in the final phase. The results are interpreted as supporting preparatory response and conditioned aversiveness interpretations of some of the phenomena in signaled shock studies.
\end{abstract}

Numerous studies have reported a preference for signaled shock, but a few studies have reported a failure to find a preference for signaled shock (see Badia, Harsh, \& Abbott, 1979, for a review). One important factor appears to be whether the shock is unscrambled and modifiable or relatively unmodifiable through the use of scrambled footshock or fixed electrodes. Those reporting a preference have used unscrambled footshock (e.g., Lockard, 1963), scrambled footshock (e.g., Arabian \& Desiderato, 1975), or fixed electrodes (e.g., Miller, Marlin, \& Berk, 1977). Those reporting no preference have used only scrambled footshock or fixed electrodes (e.g., Biederman \& Furedy, 1973; Crabtree \& Kruger, 1975).

In all of the studies on signaled shock, individual rats have experienced only one type of shock: unscrambled, scrambled, or fixed-electrode shock. For the present study, the threefold plan was, first, to establish a preference for signaled unscrambled shock using essentially the same procedures used by Lockard (1963), second, to establish the reliability of the effect by reversing the relation between signaled vs. unsignaled shock and the correlated stimulus dimensions (light vs. dark and stripe vs. no stripe), and third, to assess the effect of changing from unscrambled to scrambled shock.

The purpose of changing the type of shock was to

Requests for reprints should be sent to Brian M. Kruger, Department of Psychology, Wright State University, Dayton, Ohio 45435 . pit one theoretical interpretation (the preparatory hypothesis) against two other interpretations (the information hypothesis and the safety hypothesis). If the preference for signaled unscrambled shock is due solely to the opportunity to prepare for and modify shock by postural adjustments, then changing to scrambled shock should diminish or eliminate the preference, since postural adjustments should be less effective in modifying the shock. If signaled shock is preferred because of the information concerning time of shock, regardless of modifiability, then changing to scrambled shock should have no effect. Similarly, if signaled shock is preferred because the absence of the signal indicates a shock-free or safe period, then changing to scrambled shock should have no effect. (See Badia et al., 1979, for an overview of the major theoretical interpretations.)

\section{METHOD}

\section{Subjects}

The subjects were four female Long-Evans hooded rats, ranging in age from 130 to 150 days. All of the rats had been previously used as subjects in two studies in an appetitive runway. They were individually caged and maintained with free access to Purina Lab Chow and water throughout the experiment.

\section{Apparatus}

The translucent white plastic shuttlebox was located in a dark sound-reduction room with 67- $\mathrm{dB}$ white-noise background and was maintained at $296^{\circ} \mathrm{K}$. The $2.4-\mathrm{mm}$-diam stainless steel floor grids were spaced every $1.3 \mathrm{~cm}$. During scrambled shock sessions, vertical grids spaced every $6.4 \mathrm{~mm}$ covered the walls of each $8.1 \times 28.4 \times 20.3 \mathrm{~cm}$ chamber. The chambers were separated 
by a 16-mm-thick aluminum plate for forced enclosures. For choice sessions, a plate with a $9.2-\mathrm{cm}$ high by $7 \mathrm{~cm}$ wide doorway was employed; the threshold, elevated $2.5 \mathrm{~cm}$ above the grid floor, served as a hurdle between chambers. Two horizontal infrared beams located $3.2 \mathrm{~cm}$ above the floor and 2.5 and $7.6 \mathrm{~cm}$ inside the door end of each chamber were used in sensing the rat's location.

All shocks were of $2 \mathrm{sec}$ duration. Unscrambled shock was $85 \mathrm{~V}$ ac, presented via a $10 \mathrm{~K}$ series resistor with opposing momentary polarities present on alternating floor grid bars; the vertical plates were connected to one output of the shock source. Scrambled shock was delivered by an I-O-Tek Model 101 shock scrambler. The momentary potentials were ground for one output and $+110 \mathrm{~V} \mathrm{dc}$ via $47 \mathrm{~K}$ series resistors for the other eight outputs. Each of the eight outputs connected to the floor grids was connected to every eighth bar. All vertical grids and plates were connected to the ninth output of the scrambled shock source. Polarity changes occurred sequentially at the rate of 120 changes $/ \mathrm{sec}$.

The $1,000-\mathrm{Hz}$ tone preshock signal at $77 \mathrm{~dB}$ was presented via a speaker located behind the rear wall of the signaled shock chamber. Signals terminated with shock onset. Shock interpresentation intervals varying from 60 to $180 \mathrm{sec}$ in $20 \mathrm{sec}$ intervals were block randomized. The interstimulus interval was $4 \mathrm{sec}$ for $25 \%$ and $8 \mathrm{sec}$ for $75 \%$ of the presentations in the signal chamber for Weeks $1-29$, and $8 \mathrm{sec}$ for $100 \%$ of the presentations for Weeks 30-33. Two stimulus dimensions were correlated with signal location. In one chamber, a 1.9-cm-wide
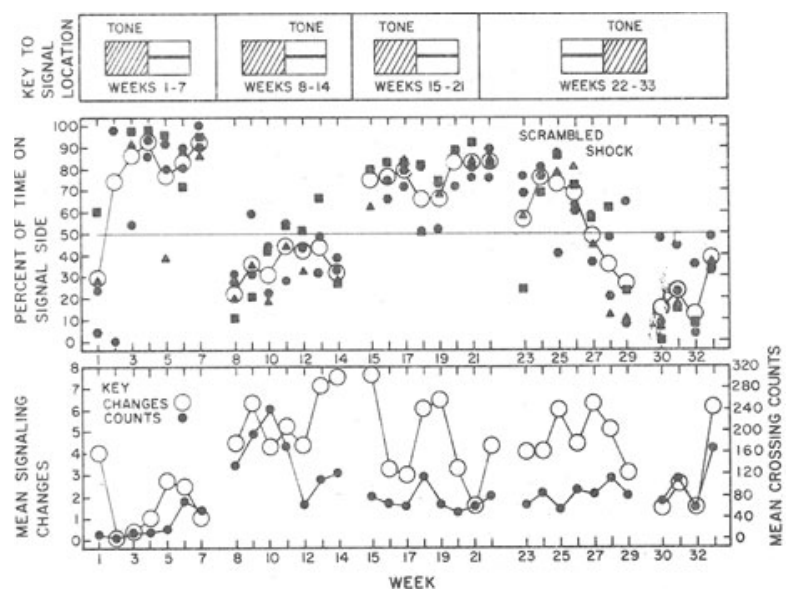

Figure 1. Data for Rat 1.
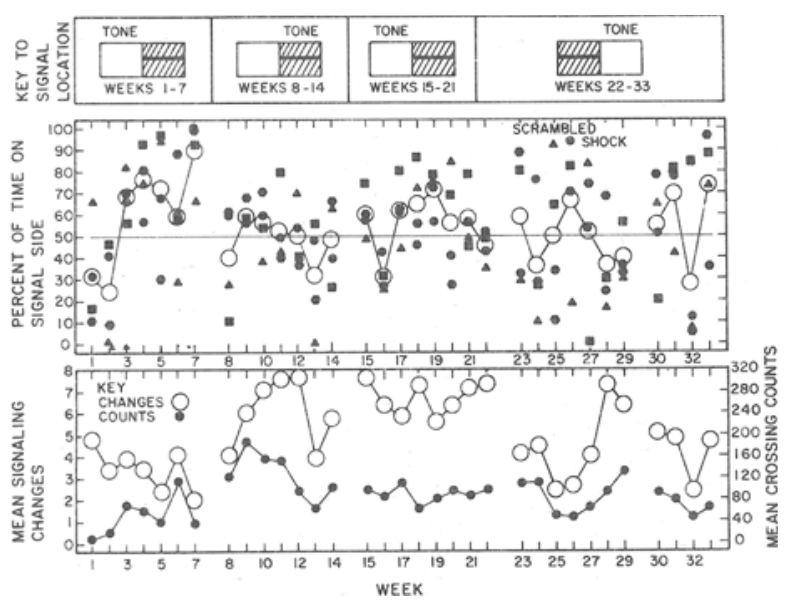

Figure 3. Data for Rat 3. black stripe was centered lengthwise on the white subfloor $2.5 \mathrm{~cm}$ below the floor grids. One chamber was illuminated at $18.31 \mathrm{x}$, and the other darkened chamber received its sole illumination from ambient light passing through the doorway.

\section{Procedure}

Prior to each 1-h free-choice session, each rat was enclosed in each side of the shuttlebox for $30 \mathrm{~min} / \mathrm{side}$, beginning with the signaled side. Each rat received a 3-day break between succeeding sets of four daily sessions.

The study was conducted in six phases of $7,7,7,1,7$, and 4 weeks, respectively, for a total of 33 weeks and 132 sessions. Unscrambled shock was used for all phases except the fifth, for which scrambled shock was used.

A successive reversal design was used for the first three phases. During Phase 1, half of the rats received the signal on the illuminated side and half on the darkened side. For Phase 2, the correlations for individual rats between signal vs. nonsignal and light vs. dark were reversed, and they were reversed again for Phase 3. During Phases 1-3, all stimulus locations remained constant for each rat, except for the changes in tone location. The stripe was located on the (arbitrarily designated) right side of the apparatus. For half the rats, the left side was illuminated; for the other half, it was darkened. The tone signal was located on the left side during Phases 1 and 3. (These relations are illustrated in the top sections of Figures 1, 2, 3, and 4.)

In order to determine the extent to which spatial location cues other than stripe and illumination level, if any, controlled

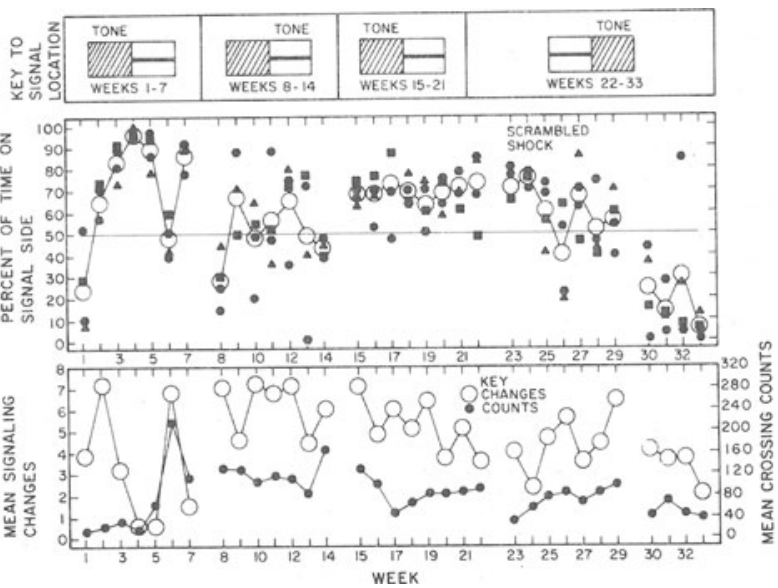

Figure 2. Data for Rat 2.

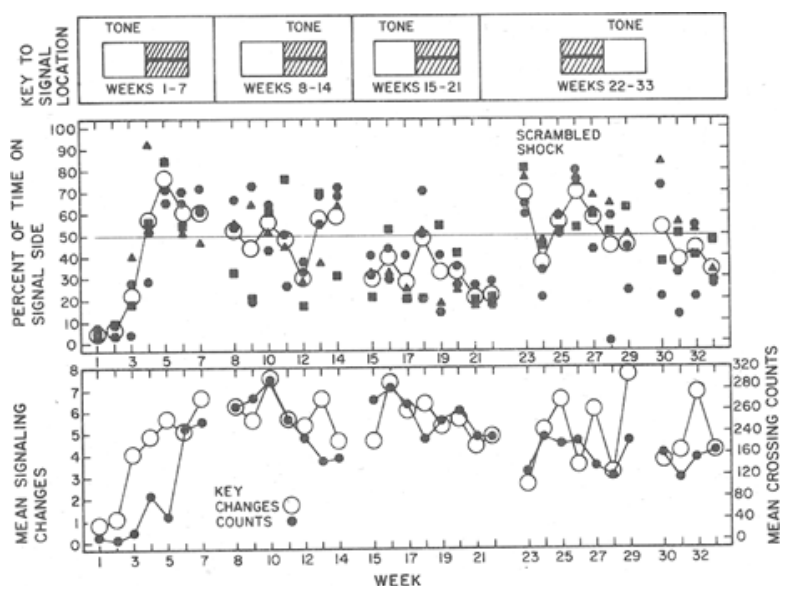

Figure 4. Data for Rat 4 
choice behavior, all stimuli (tone, stripe, and illumination levels) were changed from left to right or vice versa for Phase 4 . These locations were maintained for the remainder of the experiment. In summary the correlations for individual rats between tone/no tone, stripe/no stripe, and light-dark were the same throughout the experiment, except that the relations between the signal and the two correlated stimulus dimensions were reversed during Phase 2.

The number of seconds on the signal side were counted for each session and linearly transformed to percentages of total time. (A rat mostly on the signal side was considered on the signal side as long as the first beam inside the nonsignal side was not obstructed.) At the time for each scheduled signal onset, the decision to present the warning signal required that the rat be on the signal side. A rat's breaking a beam on the nonsignal side during signal presentation did not result in signal termination.

Two measures of activity were recorded. The number of crossings per session were counted. A crossing was defined as beginning when beams in only one chamber were concluded and as ending when beams in only the other side were interrupted. Signaling changes were also counted. A signaling change occurred when only one of two successive shocks was signaled. Weekly per-session averages were computed for all three measures.

\section{RESULTS}

During Week 1, all rats spent more time on the nonsignal side than on the signal side. Their percent time in the signaled shock side of the shuttlebox was significantly below random $(50 \%)$ performance $[t(3)=4.53$, $\mathrm{p}<.05]$. However, all rats developed a preference for signaled shock within Weeks $1-7$, with the average time on the signal side for Week 7 exceeding $80 \%$. Their percent time in the signaled shock side of the shuttlebox was significantly above chance for Week $7[\mathrm{t}(3)=4.31$, $\mathrm{p}<.05$ ]. Rats 1 and 2 , which received signaled shocks while in the dark side, developed a preference for signaled shocks more rapidly than did Rats 3 and 4, which received signaled shocks while in the lighted side (see Figures 1, 2, 3, and 4, respectively).

The top section of each figure shows the correlations among the tone/no-tone, light-dark, stripe/no-stripe, and left-right variables for each phase of the study. The large open circles in the middle section represent the (mean) percent of time spent in the signaled shock side for the four sessions each week. The daily percentages for Days 1, 2, 3, and 4 for each week are represented by the solid circles, squares, triangles, and hexagons, respectively. The weekly per-session averages for the choice activity measures are shown in the bottom section of each figure and will be described following the description of the choice data.

Reversal of the location of the tone signal with respect to the two correlated stimulus dimensions during Weeks 8-14 resulted in a drop in percent choice of the signal side to the random behavior level of approximately $50 \%$. There was no evidence of a reversal for any rat.

During Weeks 15-21, the conditions were again reversed. With the return of the original conditions, a preference for the signal side was again demonstrated by Rats 1 and 2. Neither Rat 1 nor Rat 2 showed as marked a preference as during Week 7 , although Rat 1 did exceed $80 \%$ for Weeks 20 and 21 and Rat 2 exceeded $70 \%$ for Weeks 20 and 21. Rats 3 and 4, which again experienced the signal on the lighted side, gave no evidence of a preference for the signal side. Indeed, Rat 4 slowly developed a preference for the nonsignal side, with less than $20 \%$ spent on the signal side during Week 21.

Conditions during Week 22 were the same as for Weeks 15-21, except that all stimuli were physically shifted from left to right or from right to left. As shown in Figures 1, 2, 3, and 4, performance was unaffected; Rats 1 and 2 maintained their preference for the signal side, Rat 3 remained equivocal, and Rat 4 maintained its nonsignal side preference.

Conditions remained the same for Weeks 23-29 as for Weeks 15-22, except for the substitution of scrambled shock and the addition of vertical grids on the walls of the chambers. The preference for the signal side diminished and disappeared for Rats 1 and 2, with Rat 1 demonstrating a marked preference for the nonsignal side by Week 29 . Rat 3 continued its equivocal performance around the random behavior level, and Rat 4 returned to this same equivocal performance level.

The original conditions with unscrambled shock were again reinstated for Weeks 30-33. Rats 1 and 2 failed to reverse within these 16 daily sessions. Indeed, they showed a marked preference for the nonsignal side. Rats 3 and 4 continued their equivocal behavior.

The two measures of activity show some trends that are related to the choice data. During Weeks 1-7, as percent time on the signaled side increased, there was a general increase in crossing counts for all rats. The other activity measure, however, showed opposite trends for three of the rats. Rats 1,2 , and 3 , which demonstrated marked preferences for the signaled side by Week 7 , made a large number of signaling changes during Week 1 but were making fewer signaling changes by Week 7 . Rat 4 , however, which showed only a mild preference by Week 7 , made very few signaling changes during Week 1 and continuously increased the number of signaling changes through Week 7 .

In general, both measures of activity showed some increase following the first reversal for all rats, indicating that the change in conditions increased sampling. Both measures for all rats then showed a general decline from Week 8 to Week 33, except for Weeks 23-29. The change to scrambled shock produced some evidence of increased sampling with activity measures generally being higher for Week 29 than for Week 23.

\section{DISCUSSION}

The pre-choice-forcing procedure guaranteed that, each day, each rat experienced a minimum of $30 \mathrm{~min}$ with both signaled and nonsignaled shock conditions. This procedure should have reduced the necessity for sampling of conditions through crossover behavior; however, occasional crossovers are desirable because their occurrence suggests that high percentages for one side are not due to freezing behavior. A moderate number of crossovers was generally obtained for Rats 1, 2, and 3, except 
following the first reversal. A very high number of crossover is undersirable in that the probability of spending the same amount of time on both sides is greater regardless of the rat's preference, if any. High numbers of crossovers would be expected to be associated with near-random choice behavior levels, as was obtained for Rat 4 throughout most of the study.

The preferences that were observed during the experiment seem to be explainable in terms of preparatory responses, conditioned aversiveness, and preexperimental preferences. The forcing procedure may have been responsible for the initial preference for the nonsignal side demonstrated by all rats. The forced exposure to tone-shock pairings should result in the rapid conditioning of aversive properties to the tone. However, since the unscrambled shock is potentially modifiable through postural adjustments on the grids, a preference for signaled shock may gradually develop because the signal provides a potential advantage in preparing for shock. The observed preferences at the end of Phase 1 replicated the results of studies with unscrambled shock (e.g., Lockard, 1963). The slower development of the preference for signaled shock demonstrated by rats with the signal on the light side provides evidence for a preexperimental preference for darkness, as reported by others (e.g., Miller et al., 1977).

Unlike Lockard (1963) and other previous studies, a variable interstimulus interval was used in this study. Sometimes the tone-shock interval was $4 \mathrm{sec}$, and sometimes it was $8 \mathrm{sec}$. The fact that preferences for the signaled side developed despite this variability suggests that the preparatory responses, if any, may have been general postural adjustments in preparation for the onset of shock.

The failure to obtain a reversal in Phase 2 is not readily explainable by any of the popular views and will be discussed in detail later. The reappearance of the preference for Rats 1 and 2 in Phase 3 but not for Rats 3 and 4 may relate to the fact that Rats 1 and 2 received the signal on the dark side during Phases 1 and 3. A preexperimental preference for darkness may also be involved in Rat 4's reverting to the original preference for the dark nonsignal side during Phase 3. Rat 3's preference behavior was also fluctuating toward the nonsignal side near the end of the second reversal period (Week 22).

The change to scrambled shock and the addition of vertical grids resulted in the elimination of preference for signaled shock for Rats 1 and 2. This effect should occur if the preference is based on preparatory responding and if scrambled shock is unmodifiable through overt preparatory responses. It is noteworthy that following the elimination of the preference for signaled shock with scrambled shock, Rats 1 and 2 reverted to their original preference for the lighted nonsignal side in Phase 6 , despite the reintroduction of unscrambled shock.

The failure to find a preference for signaled scrambled shock, or more precisely, the failure to maintain a preference established with unscrambled shock, contrasts with shuttlebox studies that have reported a preference for signaled scrambled shock and one study that also reported successful reversals with scrambled shock (Miller et al., 1977). These different findings are not easily reconciled.

The following post hoc analysis is directed toward a potential explanation of the present results. If the modifiability of shock is recognized early and the preference for signaled shock is based on the effects of preparatory responding, a set for coping by general posturing may be established that may later preclude coping by any other means, such as recognizing safe periods and learning to relax during those periods. The initial preference may be established in two phases: First, fear reactions, including freezing behavior, may be conditioned to the signal; second, certain posturing on the grids may be discovered that can modify shock, a process that should be facilitated by freezing in different postures in the presence of the signal. The latter process could establish an initial preference for the signal; however, once established, if the posturing is general, presence of the signal may no longer be requisite for effective modification of shock. Thus, a change in location of the signal may not produce a change in preference for correlated stimulus values (light or dark). The use of scrambled shock and vertical grids to eliminate effective coping through general posturing may eliminate the preference for the stimuli that were first correlated with the signaled side. Unless another coping mechanism is acquired, such as learning to relax during safe periods, a preference for the nonsignal side may occur due to the continued conditioning of aversive properties to the signal.

The preceding post hoc analysis also offers an explanation for some successful reversals. If brief duration (e.g., $.5 \mathrm{sec}$ ) and relatively unmodifiable scrambled shock are used to first establish a preference (Miller et al., 1977), the mechanism upon which the preference is based may be the discovery and use of safety information for relaxation. Since this coping mechanism would require continuous attention to the location of the signal, a change in location of the signal should be followed by a change in preference for correlated stimulus values.

According to the preceding analysis, the phenomena observed following a change in location of the signal may depend upon the mechanism primarily responsible for the development of the initial preference. As Badia et al. (1979) concluded in their review, there is no single principle that can account for all phenomena in studies of preference for signaled shock.

\section{REFERENCES}

Arabian, J. M., \& Desiderato, O. Preference for signaled shock: A test of two hypotheses. Animal Learning \& Behavior, 1975, 3, 191-195.

Badia, P., Harsh, J., \& Аввотт, B. Choosing between predictable and unpredictable shock conditions: Data and theory. Psychological Bulletin, 1979, 86, 1107-1131.

Biederman, G. B., \& Furedy, J. J. The preference-for-signaled shock phenomenon: Effects of shock modifiability and light reinforcement. Journal of Experimental Psychology, 1973, 100, 380-386.

Crabtree, M. S., \& Kruger, B. M. Free choice of scrambled electric shock with rats. Bulletin of the Psvchonomic Society 1975, 6, 352-354.

LOCKARD, J. S. Choice of warning signal or no warning signal in an unavoidable shock situation. Journal of Comparative and Physiological Psychology, 1963, 56, 526-530.

Miller, R. R., Marlin, N. A., \& Berk, A. M. Reliability and sources of control of preferences for signaled shock. Animal Learning \& Behavior, 1977, 5, 303-308.

(Received for publication December 29, 1980.) 